Varthakavi, Smith, Heimann-Nichols and Rose reply:

Our previous manuscript described CAML as a host restriction factor that blocks HIV-1 release (Nat. Med. 14, 641-647, 2008). We did not assess the function of CAML as an inhibitor of $\mathrm{Vpu}^{-} \mathrm{HIV}-1$ release in $293 \mathrm{~T}$ cells. The $293 \mathrm{~T}$ cells used in our laboratory did not fit the $\mathrm{Vpu}^{-}$virus 'permissive' phenotype that has been ascribed to them by Kühl et al. (Nat. Med. 16, 155-156, 2010) and others. We have since ascertained that the permissive 293T cells (a gift from W. Sundquist's laboratory (University of Utah)) show lower amounts of CAML compared to restrictive HeLa and lymphocytic cells. We also did not test CAML against HIV-2 or macaque simian immunodeficiency virus, as did Kühl et al. (Nat. Med. 16, 155-156, 2010). Rather, we compared the ability of HIV-2 Env protein and Vpu to rescue $\mathrm{Vpu}^{-} \mathrm{HIV}$ release from simian cells. In summary, we believe that the source of cells, variation across individual cell clones and other differences in the experiments in the two reports may account for the reported discrepancies.

The CAML functional data were validated in independent experiments performed in our laboratory and that of P. Spearman. Both groups continue to see an inhibitory effect of human CAML on $\mathrm{Vpu}^{-}$ mutant virus release from COS-7 cells. The precise mechanism by which CAML interferes with HIV-1 release needs to be further investigated. In this regard, we concur with the findings from P. Spearman and his colleagues that CAML does not enhance tetherin function and does not contribute to tethering mutant virus particles to the cell surface (Nat. Med. 16, 238, 2010). However, our ongoing studies in HeLa cells suggest a notable effect of CAML on HIV-1 Gag trafficking and release (V.V., E.H.-N. and K.A. Brewer, unpublished data). Our new data, which involve live imaging of cells, shows that overexpression of CAML causes a major redistribution of Gag protein to internal cellular compartments. In contrast, Gag localizes predominantly to the cell surface in cells that are depleted of CAML. Consistent with this, we have recently shown by electron microscopy that HeLa cells overexpressing CAML and infected with $\mathrm{Vpu}^{-}$mutant virus show several vesicular structures in the cytoplasm that contain virus particles. (V.V., E.H.-N. and K.A. Brewer, unpublished data). On the basis of these and other emerging data from our laboratory (V.V., E.H.-N. and K.A. Brewer, unpublished data), we propose that CAML inhibits virus release by targeting a different step in the HIV-1 life cycle than tetherin. We, therefore, stand by the original data (Nat. Med. 14, 641-647, 2008) and have repeatedly found a role for CAML in the arrest of $\mathrm{Vpu}^{-}$mutant virus release from human cells.

\section{Vasundhara Varthakavi ${ }^{1}$, Ellen Heimann-Nichols ${ }^{1}$, Rita M Smith ${ }^{1}$ \&} Jeremy Rose ${ }^{2}$

${ }^{1}$ Department of Pediatrics, Division of Infectious Diseases, Vanderbilt University School of Medicine, Nashville, Tennessee, USA. ${ }^{2}$ Department of Microbriology and Immunology, Vanderbilt University School of Medicine, Nashville, Tennessee, USA.

e-mail:vasundhara.varthakavi@vanderbilt.edu

COMPETING INTERESTS STATEMENT

The authors declare no competing financial interests.

\title{
On the origin of glioneural neoplasms after neural cell transplantation
}

\section{To the Editor:}

A major obstacle of stem cell therapy is the risk of tumor development, a challenge addressed by Jandial and Snyder in a recent 'Bedside to Bench' article $^{1}$. The writers examine our previous publication ${ }^{2}$, which describes a donor-derived brain tumor in a patient treated by experimental injection of neural stem cells; we believe their assessment underestimates the potential risks of stem cell therapy.

Jandial and Snyder question whether neural stem cells (NSCs) are at the origin of the tumor that developed ${ }^{1}$. The protocol used, similar to protocols in use at the time of treatment, is based on mitogenic growth factors and is designed to expand NSCs. Among the cultured and expanded cells given to the patient, NSCs were no doubt present, as evidenced by the documented continuous growth of the donor-derived tumors in the vicinity of the injection sites. We proved in our study by several complementary methods that the mass was composed mainly of donor-derived cells ${ }^{2}$. Even if NSCs constituted a minor fraction of the transplant, this fraction was sufficient to give rise to a tumor.

The tumor resected from our patient was diagnosed as a glioneural tumor by experienced neuropathologists. Glia and neurons, the two principal cell types comprising the central nervous system, were both represented in the tumor, and, unlike spontaneous glioneural tumors, the glial element of the subject's neoplasm was bitypic, including not only astrocytes but also ependymal cells. The cellular composition of the glioneural tumor in this case therefore strongly supports its origin from NSCs.

In agreement with our findings, Keene et al. ${ }^{3}$ recently described a donorderived tumor, arising in a patient transplanted with intrastriatal human fetal cells, with properties similar to the one we described and to the one described by Snyder and Jandial and their colleagues in Vancouver ${ }^{1}$. The glioneural tumors from all of these patients are equivalent to the teratomas that develop from pluripotent stem cells.

Although we agree with the need for the use of very pure cell populations in future studies, improved characterization by itself will not eliminate the potential for transplanted cells to give rise to tumors.

\section{Ninette Amariglio \& Gideon Rechavi \\ Sheba Medical Center, Tel Hashomer, Sackler School of Medicine, Tel Aviv University, Tel Aviv, Israel. \\ e-mail: gidi.rechavi@sheba.health.gov.il.}

\section{COMPETING INTERESTS STATEMENT}

The authors declare no competing financial interests.

1. Jandial, R. \& Snyder, E.Y. Nat. Med. 15, 999-1001 (2009).

2. Amariglio, N., et al. PLoS Med. 6, e1000029 (2009).

3. Keene, C.D., et al. A. Acta Neuropathol. 117, 329-338 (2009).
Jandial and Snyder reply:

We in no way wish to underplay concern for safety whenever one implants a cell into an organ, particularly in the central nervous system (CNS). However, the safety concerns inherent in the biology of the stem cell (as we recently detailed ${ }^{1}$ ) are not exemplified by the case presented by Amariglio et al. ${ }^{2}$. Indeed, there is no evidence that 